\title{
Asymmetric Connections, Duplicate Layers, and a Vertically Inverted Map in the Primary Visual System
}

\author{
Dale Hogan, ${ }^{1}$ Preston E. Garraghty, ${ }^{2}$ and Robert W. Williams ${ }^{1}$ \\ ${ }^{1}$ Department of Anatomy and Neurobiology, University of Tennessee, Memphis, Tennessee 38163, and ${ }^{2}$ Department of \\ Psychology, Indiana University, Bloomington, Indiana 47405
}

The achiasmatic mutation is a remarkable and rare visual system mutation carried in a line of black sheepdogs. In affected animals, the optic chiasm is missing, and each retina projects entirely to the ipsilateral hemisphere. As a result of this navigational error, maps of visual space in the lateral geniculate nucleus (LGN) have a unique structure with mirror reversals of field position across the A-A1 border. Animals also have a persistent and severe congenital nystagmus. In this report we analyze a novel variant of the achiasmatic mutation, one in which retinal axons from only one eye successfully cross midline and in which the great majority of fibers from both eyes terminate in a single lateral geniculate nucleus. The dominant optic tract contains four times as many axons as the other tract. The hyperinnervated LGN has a lamination pattern consisting of duplicate and partly interwoven layers. A multiunit mapping

Many ingenious methods have been used to discover how retinal ganglion cells contact specific target cells in the thalamus and midbrain (Blakemore et al., 1975; Constantine-Patton and Law, 1978; Chalupa and Williams, 1984). In one such experiment, Sperry (1944) disconnected and rotated eyes of frogs early in development and showed that the reformed connections could not compensate for the anomalous orientation of the eye and produce normal visually guided behavior. These frogs could not catch flies. Maladaptive visual behavior has also been shown in mammals with mutations such as the tyrosinase (albino) mutation that perturbs the development of retinal connections (for review, see Guillery, 1986; Jeffery, 1997). The most dramatic case is the achiasmatic mutation carried in a family of Belgian sheepdogs (Williams et al., 1994; Hogan and Williams, 1995; Hogan et al., 1996). Unlike normal dogs in which $80 \%$ of retinal axons cross at midline, axons in the most severely affected mutants do not cross midline. The final connections made between the retina and its principal target, the dorsal lateral geniculate nucleus (LGN), show no compensation for the initial error. Axons from the nasal side of the retina connect to the same part of the ipsilateral LGN and with the same polarity as if they had crossed. Consequently, the nucleus contains horizontally reversed maps of the visual world, stacked in alternating layers. The behavioral consequence is congenital seesaw and pendular nystagmus (Dell'Osso and

Received April 28, 1999; revised Aug. 26, 1999; accepted Sept. 8, 1999.

This work was supported by National Research Service Award EY06560 (D.H.) and National Eye Institute Grant EY06627 (R.W.W.).

Correspondence should be addressed to Dale Hogan, Department of Psychiatry and Behavioral Sciences, University of Texas Medical Branch, 301 University Boulevard, Galveston, TX 77555-0431. E-mail: dhogan@utmb.edu.

Copyright (C) 1999 Society for Neuroscience 0270-6474/99/190001-05\$05.00/0 study of visual cortex (primarily area 17 along the marginal gyrus) shows that receptive field topography and orientation selectivity are normal. The size of central binocular visual space is nearly normal and is flanked by monocular domains in the periphery. However, there is an inexplicable vertical inversion in the orientation of the cortical representation: superior fields are located rostrally, and inferior fields are located caudally. Despite a host of drastic abnormalities at all level of the visual system, from retina to cortex, this animal was behaviorally indistinguishable from normal dogs and did not have any detectable oculomotor abnormalities.

Key words: optic chiasm; visual system development; genetic mutation; axon guidance; retina; lateral geniculate nucleus; primary visual cortex

Williams, 1995; Dell'Osso et al., 1999), similar to that noted in achiasmatic humans (Apkarian et al., 1995).

Recently, we discovered an unusual sheepdog in our colony that shed further light on the establishment of central visual pathways. This animal had hemichiasma, or half a chiasm. The majority of axons from the right eye crossed midline and joined the left optic tract, but axons from the left eye extended almost exclusively into the ipsilateral optic tract. Because of this asymmetry in crossing, there was a fourfold difference in the number of axons entering the right and left optic tracts. This asymmetric misdirection was associated with a constellation unusual features in the central visual system of this mutant that included (1) duplicated layers in the hyperinnervated LGN and loss of lamination in the poorly innervated LGN, (2) a twofold difference in retinal ganglion cell numbers between the two eyes, (3) an anomalous crossed projection from retina to basal forebrain, and (4) a vertical inversion of upper and lower visual field representations in part of primary visual cortex.

Many features of the visual system of this mutant are challeng-

This article is published in The Journal of Neuroscience, Rapid Communications Section, which publishes brief, peerreviewed papers online, not in print. Rapid Communications are posted online approximately one month earlier than they would appear if printed. They are listed in the Table of Contents of the next open issue of JNeurosci. Cite this article as: JNeurosci, 1999, 19:RC38(1-5). The publication date is the date of posting online at www.jneurosci.org.

http://www.jneurosci.org/cgi/content/full/3657 
ing to explain. Anatomically, this mutant was more severely affected than mutants with the fully penetrant phenotype in which no retinal axons cross, because retinal projections to the right and left sides of the brain were grossly imbalanced. It appeared that all regions of both retinas provided a direct connection to one side of the brain, a condition that has never been achieved experimentally. Behaviorally, however, this mutant was less severely affected than an achiasmat. In contrast to the eyerotated frogs of Sperry (1944), this mutant was behaviorally indistinguishable from normal and had no oculomotor abnormalities. Despite the gross abnormality of the chiasm, the extensive and unprecedented anatomical reorganization of retinogeniculate and geniculocortical connections resulted in normal visual behavior.

\section{MATERIALS AND METHODS}

The animal was sedated with ketamine hydrochloride $(7 \mathrm{mg} / \mathrm{kg})$ and xylazine $(0.4 \mathrm{mg} / \mathrm{kg})$. Sodium pentobarbital was used to induce $(15$ $\mathrm{mg} / \mathrm{kg})$ and maintain $(3 \mathrm{mg} / \mathrm{kg} / \mathrm{hr})$ anesthesia. The eyes were protected with contact lenses, stabilized by suturing to eye rings, and focused on a tangent screen $114 \mathrm{~cm}$ from the dog's eyes. A craniotomy was made over the left visual cortex. Low-impedance tungsten microelectrodes $(1 \mathrm{M} \Omega)$ were used to record from visually responsive cells in areas 17 and 18. The positions of the area centralis and the horizontal and vertical meridia were estimated with reference to the plotted position of the optic disks $\left(\sim 20^{\circ}\right.$ central and $6^{\circ}$ inferior in normal dogs). Small electrolytic lesions were made to facilitate electrode track identification and reconstruction. At the conclusion of the experiment, the animal was killed with an overdose of pentobarbital $(40 \mathrm{mg} / \mathrm{kg}$, i.v.), and perfused transcardially with 61 of $4 \%$ paraformaldehyde. The brain, eyes, and optic nerves were dissected free from the cranium and extraocular tissues. The brain was cyroprotected in $30 \%$ sucrose and sectioned frozen at $60 \mu \mathrm{m}$ on a sliding microtome. To identify areas 17 and 18 of visual cortex, a series of one in six sections was processed for cytochrome oxidase using standard methods (Wong-Riley, 1979). The remaining sections were mounted and stained with cresyl violet to identify LGN lamination and to locate the penetrations and lesions.

To calculate the volume of the LGN, camera lucida drawings were made of 75 (right) and 95 (left) evenly spaced cresyl violet-stained sections in each LGN. The area of each drawing was measured using a Summagraphics tablet and MacMeasure custom planimetry software. Area was calibrated, multiplied by the distance between sections, and summed to estimate LGN volume. The volume of visual cortical areas 17 and 18 was calculated in the same way, using cytochrome oxidase-stained sections to identify the areal borders.

Cross sections of nerves and tracts were embedded in Spurr's resin, and photographed on a JEOL 2000 electron microscope at a magnification of $5000-7500 \times$. A distributed set of micrographs were taken that included $2-5 \%$ of the area of the optic nerves, optic tracts, the aberrant fascicle at the chiasm, and each of its branches (Fig. $1 B$ ). These were used to estimate the average density of axons. This figure was multiplied by the area of the nerve to give an estimate of axon numbers (Rice et al., 1995). Retinas were dissected free from the pigment epithelium, mounted flat as whole mounts on gelatinized slides, and coverslipped with Gelvatol to minimize shrinkage (Hogan and Williams, 1995). Retinal ganglion cells were visualized under differential interference contrast optics. Density of ganglion cells was measured at regular intervals. In the periphery, the sampling interval was $2 \mathrm{~mm}$, and the sampled area ranged from $12,500 \mu \mathrm{m}^{2}$ centrally to $125,000 \mu \mathrm{m}^{2}$ in the far periphery. In the area centralis, the sampling interval was $250-500 \mu \mathrm{m}$, and the sampled area was $1500 \mu \mathrm{m}^{2}$.

\section{RESULTS}

Our discovery of this unilateral variant of the achiasmatic mutation was unexpected. All of the achiasmatic mutants that we have studied have been readily recognizable by the prominent nystagmus and the informal observation that achiasmatic dogs are unwilling to climb stairs or jump from a height. In contrast, this 3 -year-old female would jump readily from a height of $0.5-1.0 \mathrm{~m}$. Eye movement recordings did not show nystagmus or other abnormalities (Dell'Osso and Williams, 1995; Dell'Osso et al.,

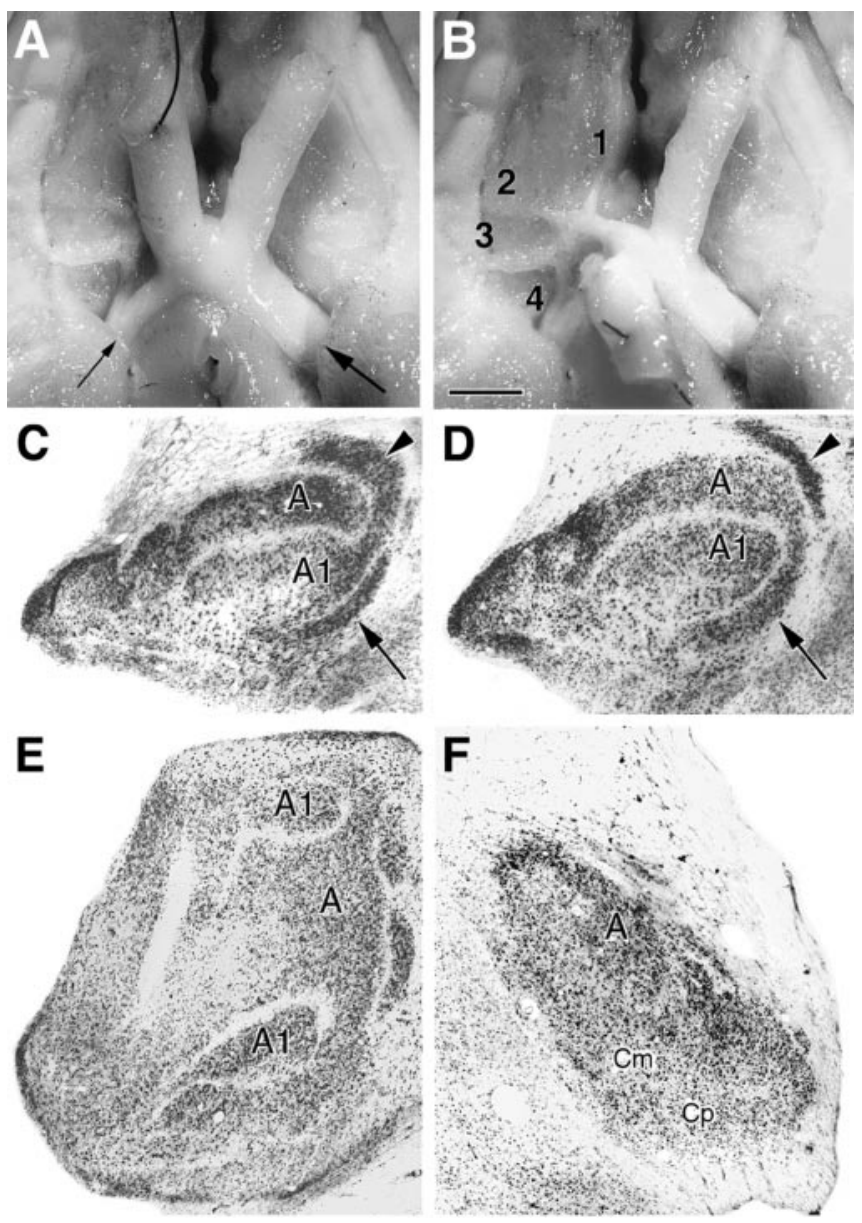

Figure 1. A, Ventral view of brain showing a unilateral chiasm defect. There is a fourfold asymmetry in the optic tracts (large and small arrows). $B$, Aberrant crossing fascicle (right optic nerve has been retracted). On the contralateral basal surface of the brain, this fascicle splits into five branches: (1) rostral branch along the olfactory tubercle, (2) lateral branch on the basal surface of the brain, (3) lateral branch that appears to enter the pyriform lobe, and (4) two caudal branches that travel ventral the optic tract. Axons from these branches did not penetrate the pia or fasciculate with the tract. $C-F$. Cresyl violet-stained coronal sections through the LGN. $C-E$, LGN on hyperinnervated (left) side. $C$. The A1 layer extended further dorsal than normal (arrowhead). There was an abnormal extra layer located medioventral to A1 (arrow). D, In this section, located $300 \mu \mathrm{m}$ rostral to the previous section, the medioventral layer (arrow) was continuous with the normal A layer and bisected the normal and dorsal A1 layers (arrowhead). E, Section through hyperinnervated LGN $\sim 1 \mathrm{~mm}$ caudal to the section in $C$. Reconstruction showed that the two layers labeled $\mathrm{A} 1$ in $E$ were continuous. $F$, Hypoinnervated (right) LGN at same levels shown in $C$ and $D$. The A, C magnocellular $(\mathrm{Cm})$, and $\mathrm{C}$ parvocellular $(\mathrm{Cp})$ layers could be distinguished, but there were no cell-sparse interlaminar zones.

1999). We assumed that this animal was a normal control. After discovering the aberrant topography in the visual cortex, more extensive assessment of this animal was carried out. However, this work was limited by the acute experimental preparation. For this reason, we do not have recordings from the right visual cortex or from either LGN.

\section{Anatomy of hemichiasma}

Essentially, this animal had "half a chiasm," or hemichiasma (Dell'Osso et al., 1999). Axons from the left optic nerve failed to extend through the optic chiasm, whereas $73 \%$ of axons in the right nerve crossed (Figs. $1 B, 2 B$; Table 1), compared with 


\begin{tabular}{lcc}
\hline Table 1. Retina and optic nerve data & \multicolumn{2}{c}{ Left } \\
\hline & Right & 589.5 \\
Retinal area $\left(\mathrm{mm}^{2}\right)$ & 591.0 & 4.8 \\
Distance AC to OD (mm) & 3.4 & 78.6 \\
Percent area nasal & 75.9 & 95,000 \\
Ganglion cells $(n)$ & 232,000 & 7,300 \\
Peak ganglion cell density (cells $\left./ \mathrm{mm}^{2}\right)$ & 8,800 & \\
Axons in nerve $(n)$ & $218,700 \pm 14,200$ & $107,800 \pm 7,600$ \\
Axons in tract $(n)$ & $59,000 \pm 7,300$ & $236,200 \pm 21,000$ \\
Axons in aberrant chiasm $(n)$ & $35,900 \pm 2,100$ & \\
Axons in rostral branch $(n)$ & $8,000 \pm 800$ & \\
Axons in lateral branch $(n)$ & $5,100 \pm 800$ & \\
Axons in pyriform branch $(n)$ & $6,800 \pm 800$ & \\
Axons in large caudal branch $(n)$ & $6,000 \pm 700$ & \\
Axons in small caudal branch $(n)$ & $1,400 \pm 200$ & \\
\end{tabular}

$80-82 \%$ in normal dogs (Hogan and Williams, 1995). A large fascicle containing 36,000 axons emerged from the left nerve at its junction with the chiasm and crossed midline dorsorostral to the chiasm (Fig. 1B). The fascicle split into five branches that extended in random directions on the surface of the basal forebrain (Figs. $1 B, 2 B$ ). Because electron micrographs revealed that the axoplasm and myelin sheaths of axons in these fascicles were indistinguishable from those of normal axons, we concluded that terminals of these axons made synaptic contacts in ectopic regions.

The areas of the retinas (Table 1 ) were the same as found in both normal and achiasmatic sheepdogs (Hogan and Williams, 1995). The peak densities of ganglion cells were in the range found for achiasmatic mutants, i.e., $\sim 25 \%$ lower than the peak density of normal sheepdogs (Hogan and Williams, 1995). Counts of axons in the optic nerves and ganglion cells in the right retina were in the range found in both normal and achiasmatic dogs, whereas the left retina contained only $50 \%$ of the normal number (Table 1). This reduction was uniform across the retina (Fig. 2A).

In coronal sections, the hyperinnervated LGN had two prominent extra layers that had cell density and composition typical of the A layers (Fig. $1 C-E$ ). One layer was situated just dorsal to the normal A layer, whereas the other was situated ventromedial to layer A1. Serial reconstruction showed that the extra dorsal layer was fused to the normal A1 layer, and the extra ventromedial layer was fused to the normal A layer (Fig. 1C,D). Despite the complex appearance of the A and A1 layers in individual sections, there was only one A layer and one A1 layer. The volume of this LGN, $58.3 \mathrm{~mm}^{3}$, was normal (Hogan et al., 1996).

The right LGN received a severely reduced innervation, mainly from the right eye. Layer A and magnocellular and parvocellular C layers could be recognized, but there were no distinct interlaminar zones (Fig. $1 F$ ). The only exception was a small area in the dorsal tail of the nucleus in which the A layers were segregated by an interlaminar zone (data not shown). This small laminated area extended only $400 \mu \mathrm{m}$ rostrocaudally and may represent input from the contralateral retina received via the caudal branches of the aberrant crossed fascicle (Figs. 1B, 2B). This LGN was truncated in the rostrocaudal dimension, and its volume was smaller than normal $\left(34.9 \mathrm{~mm}^{3}\right)$. Cell counts across all layers of the right and left LGN showed nearly identical cell densities: 17,000 cells $/ \mathrm{mm}^{3}$ in the hypoinnervated LGN versus 16,400 cells $/ \mathrm{mm}^{3}$ in the hyperinnervated LGN.

The unilateral crossing defect and the reduction in retinal
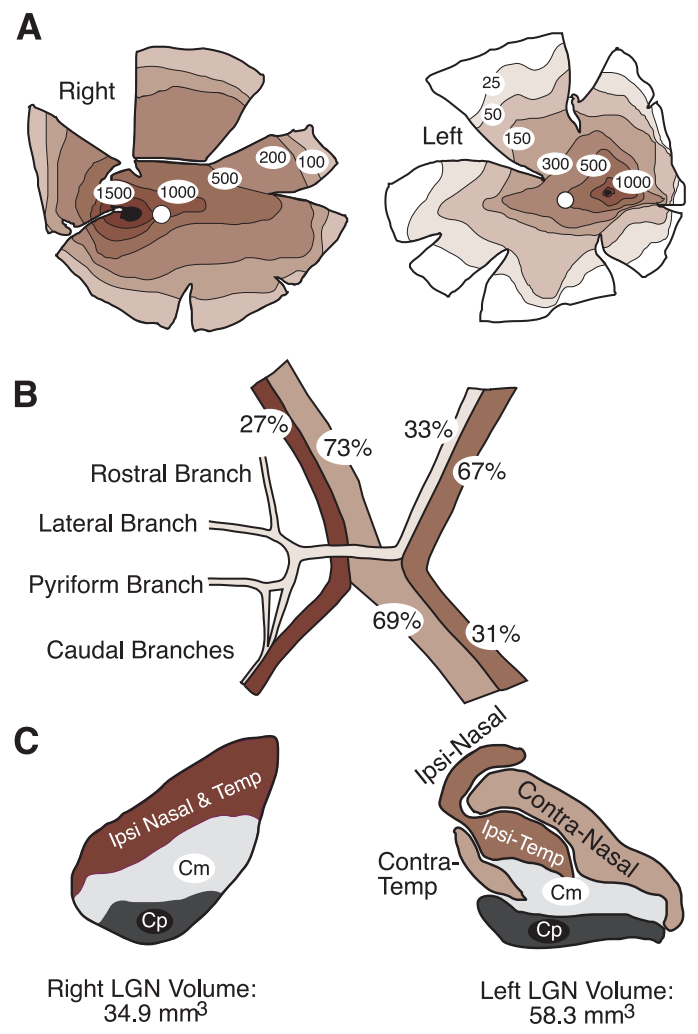

Figure 2. A, Isodensity profiles of retinas. The Right retina had a normal number of ganglion cells. The Left retina had a $50 \%$ reduction in the number of ganglion cells but a normal isodensity pattern. $B$, Schematic of chiasm and aberrant fascicles. Percentages on optic nerves and left optic tract indicate ratios of crossed versus uncrossed axons in each location. $C$, Schematics of LGNs. Target layers in the LGN are color-coded to nerve color and are labeled with the putative source of retinal input.

ganglion cells in one retina resulted in a fourfold decrease in innervation to the right LGN. This asymmetry is strongly buffered at higher levels of the visual pathway (Weliky and Katz, 1999). The hypoinnervated LGN was only $40 \%$ smaller and contained $38 \%$ fewer cells than the hyperinnervated LGN. Measurements of the volume of combined areas 17 and 18 of visual cortex showed that the "small" side and the "large" side had volumes of 1150 and $1270 \mathrm{~mm}^{3}$, respectively.

\section{Electrophysiology in the visual cortex}

We recorded from areas 17 and 18 of visual cortex in the hyperinnervated hemisphere. The most notable finding was a vertical inversion of the visual map in a large region of visual cortex (anteroposterior coordinates, P 1.8-P 5.8, along the marginal gyrus; Fig. 3). In normal cats (Tusa et al., 1978) and normal sheepdogs, upper visual fields are located caudally, and lower fields are located rostrally. In this mutant, however, upper visual fields were located rostrally, and lower visual fields were located caudally (Fig. 3). Visuotopy along the horizontal meridian was similar to that found in the cortex of the Boston-type Siamese cat (Guillery, 1986). Binocular ipsilateral fields were recorded to $10^{\circ}$ azimuth, and progression into contralateral visual space was continuous across the vertical meridian. Given the limited area of visual cortex from which we were able to record, we cannot say whether fields even more ipsilateral than $10^{\circ}$ may have existed in more lateral cortex, or whether more peripheral ipsilateral fields were suppressed. In contralateral visual space, binocular fields 
Figure 3. Visuotopy was vertically inverted in this region of primary visual cortex. Fields as high as $25^{\circ}$ were recorded in rostral areas, and fields as low as $-28^{\circ}$ were recorded $4 \mathrm{~mm}$ caudally. Binocular ipsilateral fields were recorded to $10^{\circ}$ azimuth. Monocular fields (hatched circles) were recorded in the contralateral periphsion were normal. Inset, Location of penetrations. The $17-18$ border was shifted to the lateral bank of the marginal gyrus. ery. Orientation selectivity and progres-

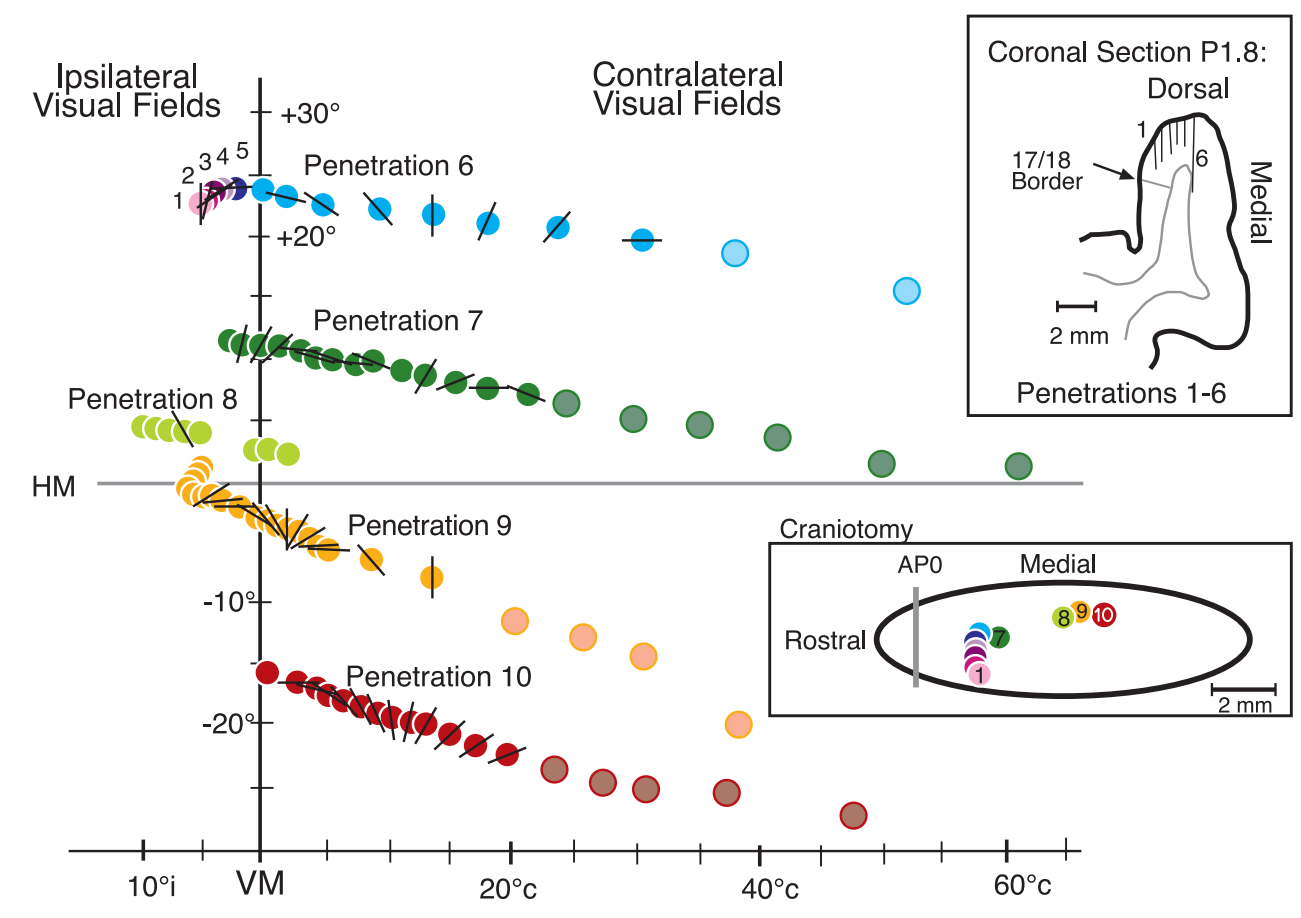

were recorded to $30^{\circ}$, the same as in normal dogs (Sherman and Wilson, 1975). Monocular fields were recorded in the periphery. The 17-18 border, normally found at the top of the marginal gyrus, was shifted to the lateral bank of the gyrus (Fig. 3). Other response properties were similar to normal dogs and other carnivores (Hubel and Wiesel, 1962; LeVay et al., 1987; Law et al., 1988). Receptive fields had robust orientation selectivity with a normal progression of orientation preference across adjacent visual fields.

\section{DISCUSSION}

\section{Reorganization of the retinogeniculate pathway}

The qualitative and quantitative changes induced by the chiasm defect are both informative and baffling when viewed in light of our current knowledge of the visual system. The extra laminae found in the hyperinnervated LGN superficially resembled the ON-OFF leaflets in the ferret (Stryker and Zahs, 1983). However, careful serial reconstruction showed that the extra A layers were extensions of normal A and A1 layers. Given what we have learned from achiasmatic and albino mutants, the organization of these layers was logical. In achiasmatic mutants, A and A1 layers innervated by the same eye fuse in the rostromedial region of the nucleus. The fact that the fused A and A1 layers were driven, respectively, by the nasal and temporal hemiretinas of the same eye was verified electrophysiologically (Williams et al., 1994; Hogan et al., 1996). Despite the fact that we did not record in the LGN of this mutant, extending the evidence from achiasmatic mutants strongly suggested that the fused layers in this unilateral mutant also received input from nasal and temporal sides of the same eye. The fact that there were two ectopic layers continuous with the normal layers suggested that this LGN received input from all four hemiretinas. Aberrant sources of input included the nasal ipsilateral projection previously seen in achiasmatic mutants and a contralateral temporal retinal projection, similar to that seen in tyrosinase mutants (Rice et al., 1995).

The random directions taken by the branches of the aberrant fascicle suggest that the retinal axons displaced onto the contralateral basal forebrain did not find cues that directed them toward the LGN. This implies that the signal directing axons to the LGN is not intrinsic to the retinal ganglion cells and is also unlikely to be a diff usible factor from the geniculate but is located within the chiasm or the anterior optic tracts (Marcus et al., 1999). In mutants without a chiasm, axons are targeted properly to the LGN, suggesting that targeting takes place in the optic tract.

In achiasmatic mutants, the number of retinal ganglion cells was equal and normal in each retina (Hogan and Williams, 1995). Therefore, the $50 \%$ reduction in the number of ganglion cells in the left retina was probably not a direct effect of the mutation. There are at least two possible explanations. First, the reduction could be attributable to increased binocular competition on the hyperinnervated side of the brain. The abnormal ipsilaterally projecting nasal ganglion cells may be at a disadvantage when forced to compete directly in the LGN for terminal space with normal contralaterally projecting ganglion cells. Intrinsic properties of LGN neurons may favor the properly crossed axons, or a delayed time of arrival at the chiasm and/or LGN may be responsible for the competitive disadvantage. Second, this result would be expected if the left optic nerve originally had a full complement of several hundred thousand axons, and the $80 \%$ of axons destined to cross did so outside the chiasm in the aberrant fascicle. Because of the lack of targeting cues outside the optic tract, the majority of these axons could not find the LGN or other sustaining postsynaptic targets, and their cell bodies subsequently died. Some mechanism may operate to reduce the survival of misdirected axons in ectopic regions. Experiments in hamsters show that misrouting of retinal axons into deafferented somatosensory and auditory thalamic nuclei caused a reduction of $76 \%$ ectopic regions (Métin et al., 1995).

\section{Is hemichiasma the same as unilateral nasal retinal ablation?}

Qualitatively, this mutant could be thought of as having an ablation of the left nasal retina. This would explain the asymmetry in retinal ganglion cell numbers between the two eyes. Norin ganglion cells, despite the availability of synaptic space in the 
mally, the temporal retina of the dog contains $\sim 50,000$ ganglion cells, with the majority projecting to the ipsilateral optic tract. In this mutant's left eye, $\sim 72,000$ axons project ipsilaterally (Fig. $2 B$ ). This increase over normal is consistent with experimental data in which elimination of competing cell populations increases the number of cells that survive the normal episode of cell death (Williams et al., 1983). This would also explain why the appearance of the hypoinnervated LGN is similar to that in carnivores that have had one eye removed early in development, because the situation is essentially identical (Garraghty et al., 1988).

Several features observed in this mutant do not fit the model of nasal retinal ablation. Most obvious is the normal distribution of ganglion cells in the left retina despite the reduced number (Fig. $2 A$ ). There is not a selective loss of cells in nasal retina. It is now obvious that the achiasmatic mutation disrupts the normal decussation pattern (i.e., nasal to contralateral or temporal to ipsilateral) in addition to the percentage of crossed axons. We previously showed that a mutant with a reduced chiasm did not have a line of decussation that was shifted to the nasal side of the retina (Hogan and Williams, 1995, their Fig. 9). Instead, there was complete overlap of the populations of ganglion cells in the ipsilateral and contralateral retinas labeled by a unilateral injection of HRP into the LGN. Data from the present case confirm that there is a profound disruption in the normal crossing pattern. The presence of two extra layers fused to the normal A layers in the hyperinnervated LGN showed that a disrupted pattern of decussation occurred even in the nerve that had a nearly normal percentage of crossed axons.

\section{Reorganization in the visual cortex}

The vertical inversion of the visual map in cortex has never been seen in a mammal. Intuitively, it seems impossible that a horizontal misdirection at the chiasm could cause a vertical defect in the cortex. One precedent for this vertical inversion can be seen in the tecta of birds. In two of seven blind-reared owls, portions of the auditory elevation map were upside-down (Knudsen et al., 1991). These maps tended to be in the most rostral and caudal parts of the tectum, and a normally oriented map was found in the central portion. In this hemichiasmatic mutant, it may be that the duplicate laminae in the hyperinnervated LGN produce duplicate maps in areas 17 and 18 of visual cortex. Like the blind barn owls, some of these maps, or some portion of a single map, may be inverted vertically. Furthermore, the visual cortex may cope with the extra input by arranging maps as a vertical inversion across a horizontal plane. Several extrastriate areas in the rat are organized in this manner (Olavarria and Montero, 1984).

In this mutant, the large asymmetry at the chiasm led to a logical reorganization of retinal axons in the LGN. However, the vertical inversion of maps in visual cortex could not have been predicted from the horizontal misrouting of retinal axons at the chiasm. Even more striking, this massive reorganization resulted in normal visual behavior. It may be that the asymmetry at the chiasm worked in favor of functional compensation. Based on our studies in the LGN of achiasmatic mutants, we hypothesized that independent maps with discordant polarity compete and cause the behavioral deficits (Williams et al., 1994). In this hemichiasmatic mutant, the relatively minor input to the right side of the brain may have been suppressed. This would result in only one functional hemisphere but no competition from discordant maps. Overall, this mutant demonstrated a remarkable and unsuspected degree of anatomical and functional plasticity in the visual system.

\section{REFERENCES}

Apkarian P, Bour LJ, Barth PG, Wenniger-Prick L, Verbeeten Jr B (1995) Non-decussating retinal-fugal fibre syndrome. An inborn achiasmatic malformation associated with visuotopic misrouting, visual evoked potential ipsilateral asymmetry and nystagmus. Brain 118:1195-1216.

Blakemore C, VanSluyters RC, Peck CK, Hein A (1975) Development of cat visual cortex following rotation of one eye. Nature 257:584-586.

Chalupa LM, Williams RW (1984) Organization of the cat's lateral geniculate nucleus following interruption of prenatal binocular competition. Hum Neurobiol 3:103-107.

Constantine-Patton M, Law MI (1978) Eye-specific termination bands in the tecta of three-eyed frogs. Science 202:639-641.

Dell'Osso LF, Williams RW (1995) Ocular motor abnormalities in achiasmatic Belgian sheepdogs: unyoked eye movements in a mammal. Vision Res 1:109-116.

Dell'Osso LF, Hogan D, Jacobs JB, Williams RW (1999) Eye movements in canine hemichiasma: does human hemichiasma exist? NeuroOphthalmol, in press.

Garraghty PE, Shatz CJ, Sur M (1988) Prenatal disruption of binocular interactions creates novel lamination in the cat's lateral geniculate nucleus. Vis Neurosci 1:93-102.

Guillery RW (1986) Neural abnormalities of albinos. Trends Neurosci 9:364-367.

Hogan D, Williams RW (1995) Analysis of the retinas and optic nerves of achiasmatic Belgian sheepdogs. J Comp Neurol 352:367-380.

Hogan D, Garraghty PE, Williams RW (1996) Lamination and visual topography in the lateral geniculate nucleus of normal and achiasmatic dogs. Eur J Anat 1:3-11.

Hubel DH, Wiesel TN (1962) Receptive fields, binocular interaction, and functional architecture in the cat's visual cortex. J Physiol (Lond) 160:106-154.

Jeffery G (1997) The albino retina: an abnormality that provides insight into normal retinal development. Trends Neurosci 20:165-169.

Knudsen EI, Esterly SD, duLac S (1991) Stretched and upside-down maps of auditory space in the optic tectum of blind-reared owls: acoustic basis and behavioral correlates. J Neurosci 11:1727-1747.

Law MI, Zahs KR, Stryker MP (1988) Organization of primary visual cortex (area 17) in the ferret. J Comp Neurol 278:157-180.

LeVay S, McConnell SK, Luskin ML (1987) Functional organization of primary visual cortex in the mink (Mustela vison), and a comparison with the cat. J Comp Neurol 257:422-441.

Marcus RC, Shimamura K, Sretavan DW, Lai E, Rubenstein JLR, Mason CA (1999) Domains of regulatory gene expression and the developing optic chiasm: correspondence with retinal axon paths and candidate signaling cells. J Comp Neurol 403:346-358.

Métin C, Irons WA, Frost DO (1995) Retinal ganglion cells in normal hamsters and hamsters with novel retinal projections. I. Number, distribution, and size. J Comp Neurol 353:179-199.

Olavarria J, Montero VM (1984) Relation of callosal and striateextrastriate cortical connections in the rat: morphological definition of extrastriate visual areas. Exp Brain Res 54:240-252.

Rice DS, Williams RW, Goldowitz D (1995) Genetic control of retinal projections in inbred strains of albino mice. J Comp Neurol 354:459-469.

Sherman SM, Wilson JR (1975) Behavioral and morphological evidence for binocular competition in the postnatal development of the dog's visual system. J Comp Neurol 161:183-196.

Sperry RW (1944) Optic nerve regeneration with return of vision in anurans. J Neurophysiol 7:57-69.

Stryker MP, Zahs KR (1983) ON and OFF sublaminae in the lateral geniculate nucleus of the ferret. J Neurosci 3:1943-1951.

Tusa RJ, Palmer LA, Rosenquist AC (1978) The retinotopic organization of area 17 (striate cortex) in the cat. J Comp Neurol 177:213-236.

Weliky M, Katz LC (1999) Correlational structure of spontaneous neuronal activity in the developing lateral geniculate nucleus in vivo. Science 285:599-604.

Williams RW, Bastiani MJ, Chalupa LM (1983) Loss of axons in the cat optic tract following prenatal unilateral enucleation: an electron microscopic analysis. J Neurosci 3:133-144.

Williams RW, Hogan D, Garraghty PE (1994) Target recognition and visual maps in the thalamus of achiasmatic dogs. Nature 367:637-639.

Wong-Riley M (1979) Changes in the visual system of monocularly sutured or enucleated cats demonstrable with cytochrome oxidase histochemistry. Brain Res 171:11-28. 